\title{
Uso de los medios sociales por las empresas líderes en telecomunicaciones de Ecuador ${ }^{\mathrm{i}}$
}

\section{Use of social media by the leading telecommunications companies in Ecuador}

\section{Uso de mídia social pelas principais empresas de telecomunicações do Equador}

\author{
Andrea Victoria Velásquez Benavides ${ }^{1}$ \\ Universidad Técnica Particular de Loja (Ecuador) \\ avvelasquez@utpl.edu.ec \\ Vanessa Karina Duque Rengel ${ }^{2}$ \\ Universidad Técnica Particular de Loja (Ecuador) \\ vkduque@utpl.edu.ec \\ Fanny Yolanda Paladines Galarza ${ }^{3}$ \\ Universidad Técnica Particular de Loja (Ecuador) \\ fypaladines@utpl.edu.ec
}

Fecha de recepción: 12 de agosto de 2020

Fecha de recepción evaluador: 16 de agosto de 2020

Fecha de recepción corrección: 20 de agosto de 2020

\footnotetext{
${ }^{1}$ Directora del Departamento de Ciencias de la Comunicación de la UTPL. Doctora en Comunicación e Industrias Creativas y Máster Universitario en Comunicación e Industrias Creativas por la Universidad de Santiago de Compostela. ORCID: https://orcid.org/0000-0003-0534-290X

2 Doctoranda en Comunicación e Información Contemporánea de la Universidad Santiago de Compostela. Especialista en Liderazgo, Cambio Climático y Ciudades en la Facultad Latinoamericana de Ciencias Sociales. Actualmente es docente titular de la Universidad Técnica Particular de Loja (UTPL). ORCID: https://orcid.org/0000-0003-0102-2829 ${ }^{3}$ Ph.D en Comunicación y Periodismo por la Universidad Santiago de Compostela de España. Forma parte del Grupo de Investigación Gestión de Comunicación Estratégica de la UTPL y de la Asociación de Investigadores en Relaciones Públicas -AIRP-. ORCID: http://orcid.org/0000-0002-1467-7501
} 


\title{
Resumen
}

En la era digital, las empresas en pro de sus intereses se han visto obligadas a adaptarse a las Tecnologías de la Comunicación e Información, haciendo uso de los medios digitales para garantizar su vigencia. En este contexto, nace este estudio, con el objetivo de analizar la gestión de comunicación estratégica 2.0 que desarrollan las principales empresas del sector Telecomunicaciones del Ecuador. Esta investigación se desarrolla en tres etapas: la primera, aplica la revisión bibliográfica para conceptualizar y contextualizar la evolución de la comunicación digital en el país y en el sector investigado; la segunda, busca describir el proceso de la comunicación digital, a través del análisis de la red social Facebook, para conseguirlo se incorporan métricas y herramientas de analítica Web; en la tercera, se analiza cualitativamente la gestión de la comunicación 2.0. a partir de entrevistas a profundidad a expertos en el área. Finalmente, se desarrolla una triangulación de información que permite describir la comunicación 2.0. y los retos del nuevo entorno digital.

Palabras clave: Comunicación 2.0.; gestión de comunicación digital; empresas de telecomunicaciones; medios sociales; Facebook.

\begin{abstract}
In the digital age, companies in pursuit of their interests have been forced to adapt to Communication and Information Technologies, making use of digital media to guarantee their validity. In this context, this study was born, with the objective of analyzing the management of strategic communication 2.0 developed by the main companies in the Telecommunications sector in Ecuador. This research is developed in three stages: the first, applies the bibliographic review to conceptualize and contextualize the evolution of digital communication in the country and in the investigated sector; the second seeks to describe the process of digital communication, through the analysis of the social network Facebook, to achieve this, metrics and Web analytics tools are incorporated; in the third, the communication management 2.0 is analyzed qualitatively. from in-depth interviews with experts in the area. Finally, a triangulation of information is developed that allows to describe communication 2.0. and the challenges of the new digital environment.
\end{abstract}

Keywords: Communication 2.0.; digital communication management; telecommunications companies; social media; Facebook.

\section{Resumo}

$\mathrm{Na}$ era digital, as empresas na prossecução dos seus interesses viram-se obrigadas a adaptar-se às Tecnologias de Comunicação e Informação, valendo-se dos meios digitais para garantir a sua validade. Neste contexto, nasceu este estudo, com o objetivo de analisar a gestão da comunicação estratégica 2.0 desenvolvida pelas principais empresas do setor de Telecomunicações do Equador. Esta pesquisa se desenvolve em três etapas: a primeira, aplica a revisão bibliográfica para conceituar e contextualizar a evolução da comunicação digital no país e no setor investigado; a segunda busca descrever o processo de comunicação digital, por meio da análise da rede social Facebook, para tanto, são 
incorporadas métricas e ferramentas de web analytics; na terceira, a gestão da comunicação 2.0 é analisada qualitativamente. a partir de entrevistas em profundidade com especialistas na área. Por fim, é desenvolvida uma triangulação de informações que permite descrever a comunicação 2.0. e os desafios do novo ambiente digital.

Palavras-chave: Comunicação 2.0.; gestão de comunicação digital; empresas de telecomunicações; mídia social; Facebook.

\section{Introducción}

Los medios sociales se han convertido en un recurso que ha trastocado las estrategias de comunicación de las organizaciones, y, por lo tanto, constituyen una herramienta imprescindible en cualquier plan de comunicación y marketing como pilar sobre las que se asienta la llamada web 2.0. El término Web 2.0 o Web social comprende aquellos sitios web que facilitan el compartir información, la interoperabilidad, el diseño centrado en el usuario y la colaboración en la World Wide Web. Web 2.0 permite a los usuarios interactuar y colaborar entre sí, como creadores de contenido.

El aprovechamiento de las bondades de las redes sociales como medio para llegar a un público más amplio y objetivo es de vital importancia para las empresas que desean seguir innovando y mantenerse en el mercado. Para Serra y Martorell (2017): “es un concepto paraguas que describe las herramientas, los servicios y las aplicaciones que posibilitan y fomentan la interacción social y la creación de contenidos por parte de los usuarios a través de la red" (p.121). Así, lo corrobora también Barrio (2017) quién señala que son plataformas de publicación de contenido, donde los usuarios toman las decisiones de los contenidos que se publican, como se clasifican y como se distribuyen.

Se puede apreciar que la comunicación es el campo que más cambios ha experimentado según los avances tecnológicos digitales asociados a la informática y a las telecomunicaciones anexadas a la Internet como el recurso interpersonal, además de los medios de comunicación clásicos. Ya no es la misma comunicación horizontal, dialógica o unidireccional, la información no es estática, los públicos ya no son los mismos y las empresas tampoco; existe mayor interacción y participación ciudadana.

Se observa, en este contexto, que las organizaciones están optando hacia la comunicación "líquida", utilizando a la Web 2.0 dentro de sus estrategias de comunicación (Arroyo y Baños, 2013). Este nuevo ecosistema, afecta en gran manera la investigación, planificación y gestión de la comunicación de las empresas.

Es en este escenario, se desarrolla la presente investigación que tiene como propósito el estudio la Comunicación 2.0 en las principales empresas del sector telecomunicaciones del Ecuador. Sobresale el hecho de que las empresas de telefonía móvil Claro y Movistar son las más relevantes. Para la realización de la investigación se establece como objetivo general: analizar la gestión de comunicación estratégica 2.0 que desarrollan las principales empresas del sector Telecomunicaciones del Ecuador. Y como objetivos específicos: 1) Identificar las marcas más influyentes en las plataformas digitales y los medios sociales utilizados por las principales empresas del sector telecomunicaciones de Ecuador. 2) Identificar los índices de comunicación 2.0 en el medio social Facebook, utilizados por las principales empresas del sector telecomunicaciones para interactuar con su público objetivo. 3) Establecer la perspectiva de gestión y retos de la Comunicación 2.0. 


\section{Marco referencial}

\section{La gestión en la comunicación estratégica de las organizaciones}

Padilla (2013) y Gómez (2008), sostienen que el impacto de Internet es relativo al de la imprenta de Johann Gutenberg. Gracias a la convergencia de este medio con las tecnologías, la cultura comunicativa se ha medicado, permitiendo contemplar un nuevo paradigma comunicativo con impacto en el devenir histórico.

Pero en este proceso, juega un rol muy importante el consumidor, así lo exponen Paladines, Gutiérrez y Gutiérrez (2020):

quien define en dónde se debe centrar una organización no es el mercado ni las tendencias, sino los consumidores y prospectos. Para lo cual debemos conocer en qué, cómo, y a través de qué medios quieren ser contactados, así como las propuestas de valor que ellos consideran importantes. Sólo así se podrá elaborar un oportuno mix de acciones para captación y fidelización (p. 133).

Para Paladines (2012):

es claro que Internet está transformando la manera en que las empresas venden sus productos y las nuevas generaciones identifican a la marca y a la empresa a través de su experiencia en la Web. De hecho, las conversaciones y recomendaciones ocupan un importante porcentaje del tráfico en Internet (p.97).

Es lo que se conoce como "boca a boca", muy utilizados por Booking y Airbnb, en donde los "prosumidores" pasan a ser "adprosumidores", porque además de comprar y consumir, evalúan y recomiendan. "Este nuevo papel que ejercen las audiencias es cada vez más exigente con las organizaciones, pues reclaman no solo ser protagonistas, sino partícipes de estas, pues están convencidas que su opinión influye en las decisiones de la empresa" (Duque y Cusot, 2020, p.96).

La figura del "prosumidor", surge como; "un internauta creativo, creador de su propia información. Si antes las organizaciones colgaban su información, en la Web de hoy son los usuarios los que realizan esta actividad, sobre todo por medio de las redes sociales" (Granda, Paladines y Velásquez, 2016, p. 220).

El nuevo modelo de comunicación bidireccional exige a las empresas el desarrollo de estrategias de comunicación que se enfoquen en establecer una relación, acercamiento y participación de los públicos. Grosso (2014) considera que, para cumplir con el propósito de este nuevo modelo, es necesario que las empresas sean cautas a la hora de generar y difundir contenidos de valor que no solamente genere impacto en los usuarios, sino también los incite a participar y a formar parte de una comunidad. Así los corroboran Paladines, Velásquez y Armijos (2019) quienes concluyen que:

La comunicación organizacional digital gira en torno a la necesidad de establecer acciones estratégicas acorde a las tendencias de las nuevas tecnologías de la información y la comunicación en la actual sociedad del conocimiento, donde se configuran las audiencias y los usuarios de una forma totalmente distinta (p. 225). 


\section{Uso de los medios sociales por las empresas y marcas}

Ya se ha manifestado que el Internet y los medios sociales han dado un giro a la comunicación. Ahora, la sociedad tiene mayor facilidad de utilizar medios digitales y dispositivos que le permiten acceder a cualquier tipo de información, establecer conexión con otras personas, o crear un espacio colaborativo para generar conocimiento, estimular la creatividad y la innovación (Tricoci, Rosenthal, Corral y Gil, 2013).

El carácter participativo e interactivo de estos nuevos medios apunta en dos direcciones: la personalización e individualización, es decir, no se trata solo de tener presencia en la red, sino de crear un contenido distinto, novedoso y exclusivo. La interactividad comunicativa implica relaciones complejas, pues toda la comunicación la ejercen seres humanos. (Rodríguez-Hidalgo, Rivera-Rogel y Velásquez, 2020, p.51)

Los encargados de gestionar la comunicación digital deben converger la habilidad y la experticia para generar estrategias y contenidos de valor que capten las nuevas necesidades de los públicos. Perfiles empresariales actuales como el Community Manager, destina estrategias y optimiza herramientas para conseguir respuesta inmediata en los públicos y aumento de tráfico de información (Hernández, Silva y Rivera, 2013). Este nuevo ecosistema de comunicación ha permito a las empresas utilizar sus herramientas para conseguir sus objetivos. Castelló (2013) sostiene que el Internet, las redes sociales y las TIC, convergen en las estrategias de las empresas, generando mensajes más personalizados, una interacción con el usuario basada en la satisfacción y estrategias de segmentación para llegar a un segmento más contundente. Ambos autores afirman que las empresas que estén dispuestas a afrontar el cambio pueden caer en la vulnerabilidad o aprovechar nuevas oportunidades.

Es importante reconocer todo lo que las empresas hacen por adaptarse, por innovar y optimizar sus esfuerzos mediante Internet y las redes sociales. Sin embargo, estas deben ser muy cautas al momento de gestionarlas, precisamente para encontrarles la riqueza, su utilidad y también sus dificultades, sobre todo, porque que son plataformas derivadas de la red, expuestas a constantes cambios (Flores, 2009).

\section{Consumo de los medios sociales}

Para Gutiérrez (2010) es notable que las redes sociales se han convertido en un espacio que permite la interacción social de las personas. De estas, dependen para satisfacer sus necesidades de comunicación, relación, aprendizaje y conocimiento. Necesidades empleadas como variables para el desarrollo de estudios que pretenden conocer el uso y consumo de los medios sociales, el Internet y el impacto de las nuevas tecnologías en las personas (Colás, Gonzáles y de Pablos, 2013).

Revisando materia, estos estudios están dirigidos a un segmento muy vulnerable a los impactos y efectos de las redes sociales; los jóvenes y niños. López, Callejo, Mendizábal y Cajiao (2013) señalan que los jóvenes y los niños son los principales segmentos de estudio por la creciente usabilidad de las tecnologías de la información y por los nuevos patrones de comportamiento en el contexto de la comunicación, más 
orientado a multimedialidad e interactividad. Por otro lado, Igarza (2010) plantea en sus estudios que el consumo de redes sociales e Internet debe ser estudiado en otros segmentos. Para el autor, este nuevo comportamiento social y la nueva forma de consumir los nuevos medios pueden ser analizados desde la evolución tecnológica y el impacto que tiene en las actividades de las personas en general y no necesariamente en los jóvenes.

El perfil digital en Ecuador ha tenido un crecimiento significativo. "Ecuador ya supera los 17 millones de habitantes y cuenta (octubre 2018) con más de 13,6 millones de usuarios conectados y con acceso a Internet, siendo el principal destino de los mismos Facebook, YouTube y Google como buscador". (Del Alcazar, 2018, s/f.)

Las investigaciones que se han planteado establecen que el consumo de redes sociales se debe principalmente por la necesidad de potenciar las relaciones personales, generar y adquirir información y para el entretenimiento. Estas variables no solamente son analizadas en la academia, sino también por empresas o grupos que pretenden conocer cada vez más a sus públicos y así posicionar a su producto o marca.

\section{Principales empresas de Telecomunicaciones en Ecuador}

La recesión económica que atravesó el Ecuador durante el 2016 y el primer semestre del 2017, permite dilucidar los principales sectores que se vieron afectados y sufrieron contracciones que implicaron menos ingresos y afectación en la aportación del PIB del país. Sin embargo, pese a estas contracciones económicas, existen sectores que aún mantuvieron altos niveles de ingresos y por ende de rentabilidad, por lo que es importante destacar su gestión del sector de las TIC para mantener y fortalecer la economía pese al crítico entorno. (Telégrafo, 2017)

La Computerword de la Revista Ekos (2017) presentó la edición del Ranking TIC, en donde establece información de las 50 empresas de Telecomunicaciones, de las que se tomó 10 para el presente estudio. En el informe hasta el 2017 registra en el Ecuador cerca de 1.700 empresas relacionadas con el sector tecnológico, los cuales generan ingresos alrededor del USD 1000.000 anuales. Del total de estas, el 91\% corresponde a la suma de los ingresos de las compañías que forman parte del Ranking TIC, lo que establece que existe una gran concentración de mercado en este sector.

Para la elaboración del Ranking TIC, Computerworld se contó con la información de la Superintendencia de Compañías, Valores y Seguros, que proporcionaron datos del sector societario y de mercado de valores. El Ranking se ordena de menor a mayor según el valor reportado de los ingresos totales, además de las utilidades brutas y del impuesto causado. Las telecomunicaciones constituyen uno de los sectores más influyentes e importantes que contribuyen al presupuesto general del Estado, pero en contraposición de esta realidad, se conoce que aún muchas poblaciones carecen de la tecnología global (Burgos, 2009). De ahí que el Estado debería buscar continuamente el acceso libre de toda la sociedad a las telecomunicaciones, no solo como un servicio público, sino también como un derecho para todos. En efecto, Mondría (2004), señala que la desregulación implica la modificación de poner en libertad o permitir que las telecomunicaciones no 
sean restringidas para las clases de estrato social bajo, sino que esta sea de libre acceso y libertad de escogimiento.

\section{Metodología}

En el presente estudio se utilizó una metodología de enfoque mixto, es decir, que combinó métodos cualitativos, que ofrecen "al investigador métodos y herramientas viables y confiables para hacer de una investigación una fuente de información para la toma de decisiones" (Pelekais, 2000, p.351), y cuantitativo de investigación, que "proporciona información objetiva, estadísticamente confiable, que para la mayoría puede ser relativamente fácil entender" (Pelekais, 2000, p.350).

El universo a investigar, como se mencionó anteriormente, es el sector empresarial de telecomunicaciones de Ecuador, se seleccionó como muestra a las 10 empresas más rentables país, de acuerdo con el ranking de la revista Ekos. El análisis se centra específicamente en la red social Facebook, considerada una de las redes con mayor aceptación en el país y con mayor diversidad de públicos.

Para el desarrollo, se dividió el estudio en tres etapas.

\section{Etapa 1. Contextualización de la comunicación 2.0.}

En esta etapa se realiza un estudio exploratorio, que inicia con el proceso de revisión bibliográfica, a fin de establecer un contexto de la evolución de la comunicación digital en el sector empresarial de telecomunicaciones.

\section{Etapa 2. Análisis de la comunicación estratégica 2.0}

El segundo momento de la investigación consiste en un estudio descriptivo, que busca explicar el proceso de la comunicación digital en las principales empresas del sector telecomunicaciones en Ecuador. Para conseguirlo se incorporan métricas y herramientas de analítica Web.

En esta etapa se aplica el método cuantitativo a través del modelo de evaluación de los procesos de comunicación digital y social utilizados en investigaciones previas (Túñez, Altamirano y Valarezo, 2016; Altamirano, Marín-Gutiérrez y Ordóñez, 2018)

- Modelo de Evaluación de la Comunicación 2.0 en los Medios Sociales: este modelo valora la comunicación 2.0 que se desarrolla en los medios sociales, partiendo de la premisa que este tipo de promoción debe cumplir con las características principales de la Web 2.0. La medición se realiza en base a la cuantificación de las acciones e interacciones en los medios sociales a través de parámetros como: presencia o impacto, actividad e interactividad. Este modelo se adapta a cada uno de los medios sociales en función de las características diferenciadoras que tienen. 
Adicional en esta etapa, se desarrolla un análisis de contenido, que es una técnica para estudiar y analizar la comunicación de una manera objetiva, sistemática y cuantitativa. En ocasiones se extiende la definición del análisis de contenido a una técnica de investigación para hacer inferencias válidas y confiables de datos con respecto a su contexto (Toro Jaramillo y Parra Ramírez, 2006, p. 169).

Para aplicar esta técnica, primero se identificó los tipos de contenido que generan las empresas evaluadas en el medio social Facebook lo que permitió clasificar y catalogar la información para determinar el tipo de publicidad que se publica en la red social. También, se analizó los contenidos con valor agregado que benefician al usuario con la finalidad de posicionar la marca y generar engagement. Estos datos fueron contrastados con las reacciones que los seguidores tienen en función del tipo de información que comparten

\section{Etapa 3. Perspectiva de expertos}

Finalmente, en esta etapa de caracter cualitativa se elaboran tres entrevistas a profundidad a expertos de comunicación digital, con el fin de conocer las perspectivas de gestión de la comunicación del sector empresarial objeto de estudio y los nuevos retos que plantea el entorno digital.

\section{Muestra}

El universo a investigar son las principales empresas del sector telecomunicaciones. Para realizar la selección de la muestra se utilizó como referencia el Ranking de las empresas en el país elaborado por la Revista Ekos en base de las ventas que registran en el año.

Tabla 1. Ranking Telecomunicaciones Ecuador

\begin{tabular}{lcccc}
\hline $\begin{array}{l}\mathrm{Nr} \\
0 .\end{array}$ & RAZÓNSOCIAL & $\begin{array}{c}\text { INGRESOS } \\
\text { TOTALES }\end{array}$ & $\begin{array}{c}\text { UTILIDA } \\
\text { DES }\end{array}$ & $\begin{array}{c}\text { IMPUESTO } \\
\text { CAUSADO }\end{array}$ \\
\hline 1 & CLARO & $1.446,16$ & 276,86 & 67,8 \\
\hline 2 & CNT & 934,56 & 183,45 & \\
\hline 3 & MOVISTAR & 680,83 & 60,04 & 16,73 \\
\hline 4 & DIRECTV & 166,86 & 12,53 & 4,68 \\
\hline 5 & TELCONET & 143,39 & 9,66 & 2,61 \\
\hline 6 & SERVICIOS DE TELECOMUNICACIONES & 103,04 & 15,61 & 1,95 \\
\hline 7 & CETEL S.A. & & & 2,71 \\
\hline 8 & HAWEITECHNOLOGIES CIA.LTDA. & 102,09 & 0,92 & 0,9 \\
\hline 9 & NETLIFE MEGADATOS S.A. & 54,61 & 5,52 & 0,1 \\
\hline 10 & ALCATEL-ECUADOR S.A. & 44,09 & 0,11 & 0,61
\end{tabular}

Fuente: Revista Ekos 2017. 
Se establecen en primera instancia las 10 empresas más influyentes del sector telecomunicaciones. De las cuales se toma una muestra intencional, basado en el criterio de uso de la red social Facebook con cuenta oficial para Ecuador y que pertenezcan al sector privado. Se excluyen del análisis a las empresas que están en proceso de liquidación o fusión durante el periodo de investigación. Las empresas seleccionadas para el análisis se citan a continuación: Claro, Movistar, Telconet, Netlife, Alcatel y TV Cable.

\section{Resultados}

\section{Comunicación 2.0 en los medios sociales de las principales empresas de Ecuador}

En este apartado y haciendo uso de las escalas establecidas por la Web 2.0, se midió el nivel de incidencia de los medios sociales dentro de las empresas de acuerdo con la escala propuesta en el modelo evaluación de Comunicación 2.0. que establece como: óptimo (100-80), aceptable alto (80-60), aceptable (60-40), aceptable bajo (40-20), mejorable (20-1).

\section{Facebook}

A continuación, en la tabla 2 , se muestra los resultados de análisis de los indicadores; presencia (en función del modelo PRGS (Presencia, Respuesta, Generación y Sugerencia) planteado por Interactive Advertising Bureau Spain (2016), según el cual la presencia se evalúa en función de dos variables: el número fans y el número de publicaciones generadas por la empresa), actividad, crecimiento, servicio, participación y engagement, que son la base para establecer el índice de comunicación efectiva de la muestra de empresas investigadas.

Tabla 2. Comunicación 2.0 en Facebook

\begin{tabular}{cccccccc} 
Page & Presencia & Actividad & Crecimiento & Servicio & Participación & Engagement & Total \\
Movistar & 89,5 & 7,5 & 100 & 96 & 100 & 28,8 & 70,3 \\
\hline Claro & 100 & 36,6 & 50,5 & 69 & 61,8 & 15,9 & 55,6 \\
\hline Netlife & 16,6 & 10,3 & 31 & 100 & 9 & 14 & 30,1 \\
\hline TVCable & 7,4 & 100 & 12 & 12,1 & 9 & 31,7 & 28,7 \\
\hline Telconet & 0,7 & 6,7 & 3,9 & 0 & 0,9 & 33 & 7,5 \\
\hline Alcatel & 10,3 & 2,5 & 0 & 0 & 0 & 0 & 2,1
\end{tabular}

\section{Elaboración propia}

\section{Índice de comunicación efectiva en Facebook}

Con la base de la tabla anterior se establecen los resultados globales del análisis de las empresas y su interacción en Facebook, mismo que es considerado como el índice de comunicación efectiva. En la tabla 3, se observa que la telefónica Movistar Ecuador 
se encuentra en primer lugar, con la categoría de Mejorable Alto, seguida de Claro la cual es aceptable y en tercer lugar Netlife Ecuador.

Tabla 3. Escala de las empresas

\begin{tabular}{ccc} 
Empresa & Total & Escala \\
\hline Movistar & 70 & Mejorable alto \\
\hline Claro & 56 & Aceptable \\
\hline Netlife & 30 & Aceptable bajo \\
\hline TVCable & 29 & Aceptable bajo \\
\hline Telconet & 8 & Mejorable \\
\hline Alcatel & 2 & Mejorable
\end{tabular}

\section{Elaboración Propia}

\section{Publicidad directa Facebook}

Para conocer la incidencia de los contenidos publicados en Facebook, se ha considerado datos referentes a publicidad directa e indirecta y publicidad con valor agregado; en función del número de reacciones generadas, obteniéndose los siguientes resultados:

Tabla 4. Publicidad directa en Facebook

\begin{tabular}{cccc} 
Empresa & $\begin{array}{c}\text { Publicidad } \\
\text { directa }\end{array}$ & $\begin{array}{c}\mathbf{N}^{0} \text { de } \\
\text { reacciones }\end{array}$ & $\begin{array}{c}\text { Tipo de } \\
\text { contenido }\end{array}$ \\
\hline Alcatel & 0 & 0 & 0 \\
\hline Claro & 81 & 33.904 & 95 videos \\
\hline Tv Cable & 43 & 2.246 & 30 videos \\
\hline Movistar & 9 & 29.419 & 12 videos \\
\hline Netlife & 63 & 7.735 & 25 videos \\
\hline Telconet & 25 & 475 & 8 videos
\end{tabular}

\section{Elaboración Propia}

La tabla que antecede, que muestra los datos de la publicidad directa destaca que, la empresa Claro es la que ha generado la publicidad directa que más reacciones ha propiciado en el público, seguida de Movistar. El resto de las empresas genera reacciones menos importantes.

\section{Publicidad indirecta}

Los resultados muestran que Movistar es la empresa que lidera de manera absoluta el uso de este tipo de publicidad. 
Tabla 5. Publicidad indirecta en Facebook

\begin{tabular}{cccc} 
Empresa & Publicidad indirecta & $\mathbf{N}^{0}$ de reacciones & $\begin{array}{c}\text { Tipo de } \\
\text { contenido }\end{array}$ \\
\hline Alcatel & 0 & 0 & 0 \\
\hline Claro & 2 & 0 & 95 videos \\
\hline Tv Cable & 0 & 0 & 30 videos \\
\hline Movistar & 1 & 167 & 25 videos \\
\hline Netlife & 0 & 0 & 8 videos \\
\hline Telconet & 0 & 0 & \\
\hline
\end{tabular}

\section{Elaboración Propia}

\section{Publicidad con valor agregado}

El valor agregado es la característica extra que un producto o servicio ofrece con el propósito de generar mayor valor dentro de la percepción del consumidor. Este término puede ser el factor determinante entre el éxito o el fracaso que una empresa tenga, debido a que se encarga, también, de diferenciarla de la competencia. (Merca 2.0, 2015). En cuanto a las empresas cuyos contenidos generan un mayor valor agregado, se puede destacar que quienes lideran esta lista son: Grupo Tv cable con 177 contenidos y de Claro con 83 .

Tabla 6. Publicidad con valor agregado Facebook

\begin{tabular}{ccc}
\hline Empresa & $\begin{array}{c}\text { Contenidos con } \\
\text { valor agregado }\end{array}$ & $\begin{array}{c}\mathbf{N}^{0} \text { de } \\
\text { reacciones }\end{array}$ \\
\hline Alcatel & 0 & 0 \\
\hline Claro & 83 & 38600 \\
\hline Tv Cable & 177 & 914 \\
\hline Movistar & 5 & 1381 \\
\hline Netlife & 3 & 484 \\
\hline Telconet & 8 & 192 \\
\hline
\end{tabular}

\section{Elaboración Propia}

\section{Perspectiva de expertos}

Con el objetivo de conocer la perspectiva crítica de expertos latinoamericanos en el área de la gestión de la comunicación estratégica 2.0 y los retos a los que se enfrentan las organizaciones del sector investigado, se desarrollaron cuatro entrevistas a profundidad, sobre cuatro variables relacionadas a la gestión presente y futura de la Comunicación 2.0. 
Tabla 7. Síntesis de entrevistas a profundidad con expertos

\begin{tabular}{|c|c|c|c|c|}
\hline Entrevistado & $\begin{array}{c}\text { En qué debe } \\
\text { enfocarse la gestión } \\
\text { de la } \\
\text { Comunicación 2.0. }\end{array}$ & $\begin{array}{l}\text { Tendencias de } \\
\text { gestión de la } \\
\text { comunicación } \\
2.0\end{array}$ & $\begin{array}{l}\text { Retos del nuevo } \\
\text { entorno digital }\end{array}$ & $\begin{array}{l}\text { Medición } \\
\text { efectiva del } \\
\text { rendimiento e } \\
\text { índice de } \\
\text { interacción }\end{array}$ \\
\hline $\begin{array}{l}\text { Christian } \\
\text { Espinosa } \\
\text { (Ecuador) }\end{array}$ & $\begin{array}{l}\text { En la percepción que } \\
\text { sus públicos tienen } \\
\text { del servicio y que se } \\
\text { reflejará en los } \\
\text { medios sociales. } \\
\text { Gestionar } \\
\text { adecuadamente la } \\
\text { interacción con los } \\
\text { públicos(reclamos) } \\
\text { que repercutirán en } \\
\text { la reputación digital }\end{array}$ & $\begin{array}{l}\text { Incremento de la } \\
\text { transmisión en } \\
\text { vivo, de público } \\
\text { en general. } \\
\text { Incremento del } \\
\text { uso de las } \\
\text { aplicaciones } \\
\text { como } \\
\text { herramienta para } \\
\text { consumo de } \\
\text { medios. }\end{array}$ & $\begin{array}{l}\text { Digitalización de } \\
\text { todo tipo de } \\
\text { transacción y } \\
\text { proceso, cómo } \\
\text { cambia la empresa a } \\
\text { partir de esta } \\
\text { realidad } \\
\text { Incorporar la cultura } \\
\text { digital en todos los } \\
\text { procesos } \\
\text { empresariales }\end{array}$ & $\begin{array}{l}\text { Incremento del } \\
\text { conocimiento de } \\
\text { interpretación de } \\
\text { resultados, no } \\
\text { solo de los } \\
\text { encargados de las } \\
\text { áreas de } \\
\text { comunicación, } \\
\text { sino también de } \\
\text { la alta dirección. }\end{array}$ \\
\hline $\begin{array}{c}\text { Dennis Reno } \\
\text { (Brasil) }\end{array}$ & $\begin{array}{l}\text { En los nuevos } \\
\text { usuarios, en especial } \\
\text { las posibilidades de } \\
\text { roaming. Eso ha } \\
\text { sido adoptado por } \\
\text { Claro en } \\
\text { Latinoamérica de } \\
\text { manera muy } \\
\text { eficiente, aunque los } \\
\text { servicios ofrecidos } \\
\text { por ellos no sean } \\
\text { igualmente buenos. }\end{array}$ & $\begin{array}{l}\text { Medios sociales } \\
\text { en crecimiento: } \\
\text { en el año 2017, } \\
\text { solo crecieron } \\
\text { YouTube, } \\
\text { Pinterest, } \\
\text { Instagram y } \\
\text { LinkedIn. Lo que } \\
\text { más ha crecido } \\
\text { fue Instagram, } \\
\text { que recibió un } \\
\text { incremento } \\
\text { superior a } 250 \% \\
\text { en su tráfico. }\end{array}$ & $\begin{array}{l}\text { Aprender a construir } \\
\text { contenidos } \\
\text { mediáticos bajo } \\
\text { colaboración y } \\
\text { expansión, cómo las } \\
\text { narrativas } \\
\text { transmedia. }\end{array}$ & $\begin{array}{c}\text { Lo más } \\
\text { importante es } \\
\text { buscar descubrir } \\
\text { cómo está la } \\
\text { imagen de la } \\
\text { empresa en los } \\
\text { espacios } \\
\text { virtuales. } \\
\text { Realizar } \\
\text { “auditoría de } \\
\text { imagen”, pues no } \\
\text { basta estar en la } \\
\text { red, sino también } \\
\text { estar bien en la } \\
\text { red. }\end{array}$ \\
\hline $\begin{array}{l}\text { Eduardo } \\
\text { Reinoso } \\
\text { (Ecuador) }\end{array}$ & $\begin{array}{l}\text { En la humanización } \\
\text { de las marcas, marca } \\
\text { con propósito, más } \\
\text { cercanas, más } \\
\text { humanas, y que } \\
\text { respondan al hombre } \\
\text { de marera sencilla. } \\
\text { Acompañada de } \\
\text { productos que llenen } \\
\text { de satisfacción al } \\
\text { cliente, que cubran } \\
\text { necesidades básicas } \\
\text { e importantes. }\end{array}$ & No aplica & $\begin{array}{l}\text { Constitución de } \\
\text { empresas } \\
\text { responsables, que } \\
\text { tengan capacidad de } \\
\text { respuesta a un } \\
\text { público más } \\
\text { emocional, sensible } \\
\text { y crítico. Consejo de } \\
\text { crisis digital que dé } \\
\text { respuesta a las } \\
\text { situaciones que están } \\
\text { pasando en las } \\
\text { sociedad, mercado y } \\
\text { empresas. }\end{array}$ & No aplica \\
\hline $\begin{array}{l}\text { Alejandro } \\
\text { Álvarez } \\
\text { (Argentina) }\end{array}$ & $\begin{array}{c}\text { En favorecer el } \\
\text { desarrollo de la } \\
\text { comunidad de } \\
\text { interés, con base a } \\
\text { los objetivos que } \\
\text { tiene la empresa } \\
\text { sobre esa } \\
\text { comunidad, para el } \\
\text { planteamiento de la }\end{array}$ & $\begin{array}{c}\text { Ciberseguridad, } \\
\text { fake news y los } \\
\text { criterios de } \\
\text { confiabilidad que } \\
\text { pueda arrojar } \\
\text { estos entornos. } \\
\text { Cambio cultural. } \\
\text { Filosofía digital } \\
\text { de las } \\
\end{array}$ & $\begin{array}{c}\text { Nueva forma de } \\
\text { planificación, por } \\
\text { escenarios basados } \\
\text { en la escucha activa } \\
\text { de la agenda de } \\
\text { temas y de } \\
\text { preocupaciones. } \\
\text { Desplegar toda una } \\
\text { gestión de } \\
\end{array}$ & $\begin{array}{c}\text { La clave sigue } \\
\text { estado en definir } \\
\text { objetivos } \\
\text { organizacionales } \\
\text { claros y vincular } \\
\text { toda la } \\
\text { planificación, la } \\
\text { gestión y la } \\
\text { evaluación de } \\
\end{array}$ \\
\hline
\end{tabular}




\begin{tabular}{cccc}
\hline estrategia. & organizaciones, & contenidos pensados & comunicación en \\
Seguimiento de & inversión de & y articulados para & función del \\
tendencias & infraestructura y & una multiplicidad de & impacto que \\
& capacitación. & dispositivos. & $\begin{array}{c}\text { logramos con las } \\
\text { acciones. }\end{array}$
\end{tabular}

\section{Elaboración Propia}

Los entrevistados coinciden en la importancia de la implementación de la cultura digital en todos los procesos empresariales, en vista de que los públicos digitales requieren disponer de un servicio ágil y rápido en el actual entorno digital. Además, enfatizan en la particularidad de servicio sensible que ofrece el sector de telecomunicaciones, en donde las acciones offline (servicio al cliente) repercuten intensamente en la reputación online, por lo tanto, deben centrarse principalmente en la gestión de estrategias de interacción con los públicos, centrándose en la humanización de las marcas, en donde se establezcan vínculos cercanos con los públicos.

\section{Discusión}

Lo digital ha permitido la trasgresión de las relaciones tradicionales que se tenía entre empresa y públicos cuando se ejecutaban acciones comunicativas off line. Hoy los expertos de comunicación son conscientes que el avance tecnológico ha afectado el ejercicio de la profesión dando paso a otra línea de comunicación: la online.

El nuevo modelo de comunicación exige a las empresas el desarrollo de estrategias de comunicación que se enfoquen en establecer una relación, acercamiento y participación de los públicos. A partir de esta dinámica, de comunicación bidireccional y la relación entre las marcas y usuarios, es necesario que, las empresas sean cautas a la hora de generar y difundir contenidos de valor que no solamente generen impacto en los usuarios sino también los incite a participar y a formar parte de una comunidad. El contexto actual de la pandemia covid-19, debe proponer aún más esta interacción y analizar las nuevas formas de consumo.

La Comunicación 2.0 en el Ecuador está siendo implementada; sin embargo, las empresas que sobresalen aún necesitan reforzar sus estrategias y herramientas para lograr sus objetivos de interacción y comunidad; mientras que las empresas con un bajo puntaje deberían buscar la forma de consolidar un vínculo de comunicación interactiva y participativa con sus usuarios. Dada la importancia y convergencia de los medios digitales en la sociedad actual las empresas del sector telecomunicaciones del Ecuador, deben estar a la vanguardia de la tecnología, ya que la misma les permitirá seguir ampliando sus horizontes. Las empresas de telefonía móvil tienen nuevos desafíos en la comunicación, a favor de su razón, producto-servicio, se puede aprovechar estratégicamente su condición, e investigar los nuevos procesos de consumo e interacción con sus audiencias.

Los expertos ratifican la importancia de una medición efectiva de la comunicación de las empresas y de los contenidos para la interacción con los públicos, haciendo énfasis en la "humanización de las marcas" como estrategia para incrementar los niveles de engagement con los públicos, en la era de la transformación digital de las empresas. 


\section{Conclusiones}

1. La Comunicación 2.0 gira en torno a la necesidad de establecer acciones estratégicas acorde a las tendencias de las nuevas tecnologías de la información y la comunicación, en la actual sociedad del conocimiento, donde se configuran las audiencias y los usuarios de una forma totalmente distinta a sus predecesoras. El índice de comunicación efectiva de las principales empresas del Ecuador, en el medio social Facebook, a pesar de ser positivo, no logra su total efectividad; puesto que los índices de algunas compañías analizadas siguen siendo escasos en las métricas propuestas, ya y no generan contenido sobre sus distintas actividades.

2. La comunicación estratégica 2.0 que desarrollan las principales empresas del sector Telecomunicaciones en Ecuador ha evolucionado a través de los medios sociales y su gestión, dejando de ser marcas convencionales y acercándose con sus públicos objetivos. Las empresas deben implementar estrategias 2.0: crear comunidades, producir experiencias y personalizar a la marca. Las marcas más influyentes en las plataformas digitales y los medios sociales utilizados son Claro y Movistar, debido a la constante adaptación que tienen en sus estrategias, herramientas y contenidos para la gestión de sus Webs y medios sociales.

3. Los contenidos generados en los medios sociales por parte de las empresas son de tipo publicitario, los mismos que son directos, indirectos, están relacionados con la empresa y promueven su marca, generando valor agregado a las mismas. En este punto al igual que en los índices de comunicación, las empresas que sobresalen en Facebook son: Claro y Movistar.

4. Como estrategia de publicidad a través de la Comunicación 2.0 las empresas de telecomunicaciones deberían implementar programas y proyectos que les involucren con la comunidad; ya que generalmente el público muestra afinidad con las empresas que manifiestan su responsabilidad con la sociedad, medio ambiente, apoyo a causas sociales y acciones que inviertan esfuerzos y recursos contra problemáticas sociales.

5. El contenido es, sin duda, la palanca que induce a que el usuario se interese y amplíe el alcance entre sus contactos; por lo tanto, las empresas deben ser más creativas en cuanto a ellos, puesto que la dinámica de estos en los medios sociales determina la aceptación que una marca tenga y la posibilidad de crecer constantemente.

\section{Bibliografía}

Arroyo, I, y Baños, M. (2013). La eficacia de la comunicación de las organizaciones del Tercer Sector en los vídeos emitidos a través de YouTube/Non profit 
communication effectiveness in the YouTube videos. Historia y comunicación social, 18, 615.

Barrio, J. (2015). La influencia de los medios sociales digitales en el consumo. (Tesis doctoral). Disponible en https://eprints.ucm.es/42339/1/T38702.pdf

Burgos, E. y Cortés, R. (2009): Iníciate en el marketing 2.0. Los social media como herramientas de fidelización de clientes. España: Gesbiblo, S. L.

Castelló, A. (2013). Estrategias empresariales en la Web 2.0. Las redes sociales online. Editorial Club Universitario.

Colás, P., González, T., y de Pablos, J. (2013). Juventud y redes sociales: Motivaciones y usos preferentes. Revista Comunicar, 20 (40), 15 -23.

Computerworld-Ekos (2017). Raking 50 empresas de Telecomunicaciones del Ecuador. Recuperado de: http://computerworld.com.ec

Del Alcazar, J. (24 de octubre de 2018). Estado Digital Ecuador - Estadísticas Redes Sociales, Aplicaciones y Sitios Web Ecuador [Mensaje en un blog]. MENTINNO, Innovation \& Lifetime Value Partners. Recuperado de: https://blog.formaciongerencial.com/estadodigitalecuadoroctubre2018/

Duque, V.K., Cusot, G. (2020). Reputación Organizacional: Fidelizando a las nuevas audiencias. En Velásquez, A., Reno, D. y Estrella, S. (Ed.), Tendencias de la comunicación II (pp. 85- 115). Madrid, España: Dykinson.

Flores Vivar, J. M. (2009). Nuevos modelos de comunicación, perfiles y tendencias en las redes sociales. Comunicar, 17(33). Recuperado de: http://www.redalyc.org/html/158/15812486009/

Gómez, J. (2008). Internet y la palabra: Un nuevo paradigma comunicativo en la cultura y la educación del siglo XXI. Puertas a la lectura, (20), 170-183.

Granda, C., Paladines, F., y Velásquez, A. (2016). Digital strategic communication in Ecuador's public organisations Current state and future projection. Revista Latina de Comunicación Social, 71, 211-231.

Grosso, C. y Lacasa, I. (2014). La adaptación de las marcas a las redes sociales. Un análisis socio-relacional de la actividad comunicativa de las marcas de refrescos en Facebook. (Tesis doctoral). Universidad Internacional de Catalunya, España.

Gutiérrez, I. (2011). Reseña de "Aprendizaje con redes sociales: Tejidos educativos para los nuevos entornos" de Castañeda Quintero, L. (Coord.). Pixel-Bit. Revista de Medios y Educación, (39), 225-226.

Hernández, A., Silva, D., y Rivera, E. (2013). El community manager: características y funciones básicas. Revista de Psicología y Ciencias del Comportamiento de la UACJS: RPCC-UACJS, 4(2), 67-75. 
Igarza, R. (2010). Nuevas formas de consumo cultural: Por qué las redes sociales están ganando la batalla de las audiencias. Comunicação Mídia e Consumo, 7(20), 5990.

La economía del Ecuador se contrajo 1,5\% en 2016. (12 de abril de 2017). El Telégrafo, $\mathrm{s} / \mathrm{f}$.

López, D., Mendizábal, I. R., Cajiao, E., y Callejo, G. (2014). Consumo de Internet en el Ecuador entre los años 2010 y 2012: hacia una ecología de la comunicación. Revista ComHumanitas, 4(1), 31-45.

Mondría, J. (2004). Diccionario de la comunicación comercial. España: Ediciones Díaz de Santos.

Padilla, D. (2013). Tendencias innovadoras en modelos comunicativos. Vivat Academia (125), 130-131.

Paladines, F y Otero, M. (2012). Gestión de la comunicación de la marca en las redes sociales: Estudio de tres casos de campañas con Facebook en Ecuador. (Tesis doctoral) Universidad Santiago de Compostela, España.

Paladines, F., Velásquez, A., y Armijos, N. (2019). Comunicación organizacional digital en las empresas del sector automotor de Ecuador. Revista Ibérica de Sistemas y Tecnologías de Información. (E26), 215-228.

Paladines, F., Gutiérrez, P., y Gutiérrez, R. (2020). El modelo de negocio y el futuro de la publicidad. La publicidad convencional y no convencional: Una revisión de las categorías. En Velásquez, A., Reno, D. y Estrella, S. (Ed.), Tendencias de la comunicación II (pp. 117- 144). Madrid, España: Dykinson.

Pelekais, C. (2000). Métodos cuantitativos y cualitativos: diferencias y tendencias. Telos, $2(2), 347-352$.

Rodríguez- Hidalgo, C., Rivera-Rogel, D., y Velásquez, A. (2020). Las audiencias en el contexto digital. En Velásquez, A., Reno, D. y Estrella, S. (Ed.), Tendencias de la comunicación II (pp. 45- 55). Madrid, España: Dykinson.

Serra, C. y Martorell, C. (2017). Los medios sociales como herramientas de acceso a la información en la enseñanza universitaria. Revistes Científiques de la Universiat de Barcelona. (32), 118-129. Disponible en https://revistes.ub.edu/index.php/der/article/view/17696

Tricoci, G. A., Rosenthal, A., Corral, P., Y Gil, P. (2014). Una mirada sobre el impacto del uso de las redes sociales en las empresas argentinas. Fórum Empresarial, 2(1), $1-7$. 


\section{Notas}

i Esta investigación se desarrolló dentro de las líneas de investigación del Grupo Comunicación Organizacional Estratégica del Departamento de Ciencias de la Comunicación de la Universidad Técnica Particular de Loja y del Observatorio de Comunicación UTPL. Agradecemos el apoyo de Pablo Daniel Tandazo Azuero, estudiante de Relaciones Públicas de la UTPL y de todos quienes contribuyeron con la información necesaria.

ii Andrea Victoria Velásquez Benavides es Directora del Departamento de Ciencias de la Comunicación de la Universidad Técnica Particular de Loja. Profesora de Imagen Corporativa, Auditoría en Comunicación y, Recepción consumo y uso de medios digitales. Doctora en Comunicación e Industrias Creativas por la Universidad de Santiago de Compostela - España y Máster Universitario en Comunicación e Industrias Creativas por la misma Universidad. Diploma Superior en Gerencia Estratégica de Mercadeo, Diploma Superior en Comunicación Corporativa. Sus publicaciones incluyen temas relacionados con Consumo y uso de medios de comunicación, competencias mediáticas, millennials, influencers, youtubers, publicidad y marca digital. Es Miembro de la red Alfamed y del Medialab UTPL.

iii Vanessa Karina Duque-Rengel es Doctoranda en Comunicación e Información Contemporánea de la Universidad Santiago de Compostela. Especialista en Liderazgo, Cambio Climático y Ciudades en la Facultad Latinoamericana de Ciencias Sociales. Magíster en Dirección de Comunicación Empresarial e Institucional por la Universidad de las Américas. Certificado Superior en Gestión del Talento Humano por la Universidad Técnica Particular de Loja Licenciada en Ciencias de la Comunicación Social por la Universidad Nacional de Loja. Cuenta con diez años de experiencia como comunicadora corporativa, consultora y periodista radial. Actualmente es docente titular de la Universidad Técnica Particular de Loja. Trabaja e investiga en el ámbito de la radio, comunicación corporativa y responsabilidad social de los medios de comunicación y el cambio climático.

iv Fanny Yolanda Paladines Galarza es Ph.D en Comunicación y Periodismo por la Universidad Santiago de Compostela de España.Forma parte del Grupo de Investigación Gestión de Comunicación Estratégica de la UTPL y de la Asociación de Investigadores en Relaciones Públicas -AIRP-. Ex Directora del Área de Marketing de la UTPL, miembro del equipo de calidad de las titulaciones de Relaciones Públicas y Comunicación Social de la UTPL. Actualmente Coordinadora de la Titulación de Relaciones Públicas. Investiga y escribe en revistas científicas Scopus, ISI, Latindex, libros, divulgativos y más, temas relacionados con la marca/branding, marca digital, gestión de la marca en redes sociales, publicidad, marketing, comunicación estratégica/corporativa; comunicación tradicional y digital, comunicación política, turística, de crisis, etc. 